\title{
Radio Access for Future 5G Vehicular Networks
}

\author{
Barbara M. Masini \\ CNR \\ IEIIT \\ Bologna, Italy \\ Email: barbara.masini@ieiit.cnr.it
}

\author{
(Invited Paper) \\ Alessandro Bazzi \\ CNR \\ IEIIT \\ Bologna, Italy \\ Email: alessandro.bazzi@ieiit.cnr.it
}

\author{
Enrico Natalizio \\ Sorbonne universités - UTC \\ CNRS, Heudiasyc UMR 7253 \\ Compiègne, France \\ Email: enrico.natalizio@hds.utc.fr
}

\begin{abstract}
Current wireless access technologies cannot satisfy all the requirements of future vehicular networks, and $5 \mathrm{G}$ could provide a solution to the actual limitations enabling super-fast, reliable, and low latency connections. The road to $5 \mathrm{G}$ vehicular networks can be considered opened by the Release 14 of Long Term Evolution (LTE), which enables vehicle-to-vehicle (V2V) communications also out of coverage of the eNodeB. This work firstly focuses on radio access of actual and next generation vehicular networks, by highlighting the main limitations and potentialities. Then, it focuses on beaconing for cooperative awareness and compares the performance of LTE when adopted in different fashions, from the actual legacy solution in vehicle-toinfrastructure (V2I) mode to future potential alternatives, such as LTE-V2V, LTE-V2V with short subframe and LTE-V2V with full duplex (FD) radios. Performance results are provided in terms of medium access efficiency and success probability of resource allocation, and show the potential advantages of future solutions, especially those provided by LTE-V2V with FD radios.
\end{abstract}

\section{INTRODUCTION}

We are assisting at a new industrial revolution, where information is a commodity taken for granted. This is even true for travelers and drivers of future connected and autonomous vehicles, who expect to know the best route, with the best comfort and in total safety.

Major advances have been made in the field of connected vehicles: the automotive and transportation industries will bring to market autonomous and connected vehicles by 2020 [1]. In the meanwhile, car makers are working to improve safety and comfort: Volvo XC60 relies on an automatic collision avoidance system which allowed in Sweden a $45 \%$ reduction in rear-end frontal crashes [2]; Tesla autopilot made (partially) autonomous driving a reality; Audi connect integrates the on-board systems and let them cooperate to provide the best comfort while traveling; etc.

In spite of all these steps ahead, still something remains unsolved to fulfill safe and comfortable traveling experiences: direct communication between vehicles. Only when vehicles will talk to each other directly sharing their own vision of the environment, an important degree of safety, autonomy, and efficiency could be granted.

If until a couple of years ago, the only available technology for vehicle-to-vehicle $(\mathrm{V} 2 \mathrm{~V})$ communication was wireless access in vehicular environment (WAVE)/IEEE 802.11p in the US [3], [4] and ETSI ITS-G5 in Europe, the new Release 14 of long term evolution (LTE), hereafter LTE-V2V and the anticipated arrival of $5 \mathrm{G}$ opened the way to new challenging possibilities [5]-[7]. Due to the wide diffusion and coverage of cellular systems and for its low latency, LTE-V2V could supplant WAVE/IEEE 802.11p (or ETSI ITS-G5) both for safety applications, traffic management and infotainment, and represents the road toward $5 \mathrm{G}$ vehicular networks.

But if WAVE/IEEE 802.11p (ETSI ITS-G5) has been widely investigated, not only in the scientific literature, but also through densely populated testbed worldwide, LTE-V2V is still in a preliminary phase. Hence, from one hand, proponents of WAVE/IEEE 802.11p (and ETSI ITS-G5) point out that these standards can satisfy all necessary vehicle-to-everything (V2X) communications in modules that are already commercially available and, from the other hand, automakers ought to wait to run cars on next generation cellular networks [8], [9].

Performance of LTE-V2V have been recently studied in the literature: in [10], an autonomous resource selection scheme for urban V2V communication scenario is presented; in [11], [12], MIMO and cooperative communications are exploited to improve the performance of $\mathrm{V} 2 \mathrm{~V}$ links.

Beaconing and selective broadcasting for cooperative awareness are addressed in [13]-[16] as the basis for all those application built on the awareness of the environment.

In this paper, we firstly provide a short survey on the initial steps toward 5G vehicular networks, addressing the standards evolution toward $5 \mathrm{G}$, the potential wireless access methodologies and the novel requirements. Then, we address beaconing for cooperative awareness and we compare the performance of LTE when adopted in different modes, from actual legacy solution toward potential 5G fashions. Specifically, we consider: i) LTE in vehicle-to-infrastructure (V2I) mode, ii) LTE in V2I mode with evolved multimedia broadcast and multicast service (eMBMS), iii) LTE-V2V, iv) LTE-V2V with short subframe and v) LTE-V2V with full duplex (FD) radios.

The paper is organized as follows: in Section II, the road to $5 \mathrm{G}$ vehicular networks is briefly described from the perspective of standards evolution; In Section III, requirements foreseen for next generation vehicular networks are reported from the point of view of different standardization entities; in Section IV new 5G vehicular network requirements and radio access techniques are described; in Section V the application of cooperative awareness is addressed and output figures for technologies comparison are described, providing example 
results. At last, in Section VI our conclusion is drawn.

\section{StAndARDiZATION EFFORTS TOWARD 5G}

Direct connectivity between vehicles has been firstly enabled by IEEE $802.11 \mathrm{p} / \mathrm{ITS}-\mathrm{G} 5$ that represented the standard de facto for $\mathrm{V} 2 \mathrm{~V}$ communications in the $5.9 \mathrm{GHz}$ frequency band [17]. Even if today the attention moves toward $5 \mathrm{G}$ cellular systems, it has to be recognized the huge effort to enable safe message exchange in high speed vehicular scenarios through a dedicated control channel. The advantage is that communication is direct between vehicles, since it operates without network coverage by an infrastructured network, in a fully distributed fashion.

This de facto standard is based on orthogonal frequency division multiplexing (OFDM) at the physical (PHY) layer and carrier sensing multiple access with collision avoidance (CSMA/CA) at the medium access control (MAC) layer. If, from one hand, this allows fast users connection and distributed resource allocation, on the other hand it does not easily scale and the performance can drastically degrade with the increase of the channel load. Hence, in highly populated scenarios, collisions may compromise the network performance in terms of throughout and latency, also for safety applications. This behaviour is even more evident if the phenomena of hidden node and capture effect occur.

New communications through the vehicles lights have been recently standardized in the IEEE 802.15.7, to allow visible light communication (VLC) even in mobile environments [18]. By exploiting VLC, vehicles can directly communicate through the front and rear lights, but only when in visibility. Hence, in spite of the simplicity of installation and the cheapness, this limits vehicular communications, where obstacles often obscure the communications. For this reason, VLC can be considered mainly as an add-on technology to be integrated and jointly adopted with other wireless access technologies toward a $5 \mathrm{G}$ scenario.

A novel solution is represented by LTE-V2V, standardized by 3GPP at the end of 2016 with the Release 14 [19], [20]. LTE-V2V allows vehicles to exchange messages directly among each other, without the intervention of the eNodeB, allowing communication also in areas without network coverage. LTE-V2V is currently designed to operate in the ITS spectrum $(5.9 \mathrm{GHz})$, in a mode to assure anonymity and no cellular subscription requirement for direct safety communications [21]. ${ }^{1}$ It is is based on the uplink PHY and MAC of LTE. Hence, it exploits OFDM at the PHY layer as IEEE 802.11p and single carrier frequency division multiple access (SC-FDMA) at the MAC layer. The use of SC-FDMA has the advantage to allow for more transmit power with the same power amplifier if compared to IEEE 802.11p; thus, longer coverage ranges or more reliable performance at the same distance can be possible. Hence, from one hand, the system is intrinsically more robust against interference if compared

\footnotetext{
${ }^{1}$ Direct communications can be supported on the in-band licensed cellular spectrum or out-of-band unlicensed spectrum. In this work, we focus only on the latter.
}

to CSMA/CA, but on the other hand resource allocation is a new issue to be faced. Optimization of the resource allocation is required both if the eNodeB pre-assigns resources to allow direct communications or if the vehicles autonomously select the resources based on a sensing phase, especially in high density and mobility scenarios. In all cases, a semi persistent scheduling algorithm is anyway applied, providing reduced signal overhead with respect to CSMA/CA, which pays the cost of the channel access contention.

Above all, the main advantage of LTE-V2V with respect to IEEE 802.11p (or even other technologies, such as VLC), is that it could address all V2X applications with a cellular technology, which is widespread and scalable, thus representing the evolution path from LTE to $5 \mathrm{G}$.

\section{Vehicular APPlications REQUiREMENTS}

Future connected vehicles will provide safer, more efficient, and more enjoyable driving experience: safer vehicles have to counteract more than 1.2 million deaths on the roads worldwide each year ${ }^{2}$, more efficient vehicles may help in saving some of the 3.1 billions gallons of fuels wasted due traffic congestion (in the US) ${ }^{3}$, and some of the $14 \%$ of all global warming from gas emissions from transportation ${ }^{4}$; more enjoyable driving experience may lead to significant societal impact.

We can distinguish three different categories of applications with different requirements: safety, traffic management, and infotainment. Safety applications require very low latency, traffic management requires real time information and the processing of short but frequent packets from a multitude of vehicles, and infotainment may require high data rate for video streaming, social gaming, etc.

In Table I, we summarize the applications foreseen by some of the main international institutions and their requirements in terms of connectivity, beacon periodicity (BP), communication range, end-to-end latency, and data rate. In particular, Table I reports the studies of ETSI and 3GPP, which represents the two main entities that have provided numerical requirements for the different applications. Requirements from ETSI can be found in [22], whereas the 3GPP working group SA1 published in [19] one of its studies.

All the applications are enabled by beacons exchange among vehicles. Beacons packets are typically short and contain basic information such as the vehicle identification, position, speed and acceleration. Hence, by exchanging beacons, vehicles become aware of the environment: more frequent beacons means a more precise knowledge of the neighboroud but higher channel load and risk of collisions. Hereafter, we call this service cooperative awareness. For example, most safety applications are guaranteed by a BP of $10 \mathrm{~Hz}$.

Thinking of future applications such as platooning, and of future self-driving cars which must autonomously and promptly react, lower latency (of the order of 1-2 ms) and very

\footnotetext{
${ }^{2}$ Global Status Report on Road Safety, World Health Organization 2015.

${ }^{3}$ Texas Transportation Institute Urban Mobility Report, 2015.

${ }^{4}$ U.S, Environmental Protection Agency (EPA) 2014
} 
TABLE I

APPLICATIONS AND REQUIREMENTS FOR ETSI AND 3GPP.

\begin{tabular}{|c|c|c|c|c|}
\hline Application & V2X & $\begin{array}{c}\text { Message } \\
\text { Type }\end{array}$ & $\begin{array}{c}\text { Beacon } \\
\text { Periodicity }[\mathrm{Hz}] \\
\end{array}$ & $\begin{array}{c}\text { End-to-End } \\
\text { Latency [ms] }\end{array}$ \\
\hline \multicolumn{5}{|c|}{ ETSI - Safety } \\
\hline Emergency electronic brake lights & V2X & Periodic & 10 & 100 \\
\hline Safety function out of normal condition warning & $\mathrm{V} 2 \mathrm{X}$ & Periodic & 1 & 100 \\
\hline Emergency vehicle warning & $\mathrm{V} 2 \mathrm{X}$ & Periodic & 10 & 100 \\
\hline Slow vehicle/Motorcycle/Road user warning & $\mathrm{V} 2 \mathrm{X}$ & Periodic & 2 & 100 \\
\hline Wrong way driving warning/Stationary vehicle warning & $\mathrm{V} 2 \mathrm{X}$ & Event-driven & 10 & 100 \\
\hline Traffic condition warning & $\mathrm{V} 2 \mathrm{X}$ & Event-driven & 10 & N/A \\
\hline Signal violation warning & $\mathrm{V} 2 \mathrm{X}$ & Event-driven & 10 & 100 \\
\hline Roadwork warning & $\mathrm{I} 2 \mathrm{~V}$ & Periodic & 2 & 100 \\
\hline Decentralized floating car data & $\mathrm{V} 2 \mathrm{X}$ & Event-driven & $1-10$ & N/A \\
\hline Overtaking vehicle warning & $\mathrm{V} 2 \mathrm{~V}$ & Periodic & 10 & 100 \\
\hline Lane change assistance & $\mathrm{V} 2 \mathrm{~V}$ & Periodic & 10 & 100 \\
\hline Pre-crash sensing warning & V2X & Event-driven & 10 & 50 \\
\hline Co-operative glare reduction & $\mathrm{V} 2 \mathrm{~V}$ & Periodic & 2 & 100 \\
\hline AcrossMerging traffic turn collision risk warning & $\mathrm{V} 2 \mathrm{~V}$ & Periodic & 10 & 100 \\
\hline Hazardous location notification & V2X & Event-driven & N/A & \\
\hline Intersection collision warning & $\mathrm{V} 2 \mathrm{~V}$ & Periodic & 10 & 100 \\
\hline Co-operative forward collision warning & $\mathrm{V} 2 \mathrm{~V}$ & Periodic/Event-driven & 10 & 100 \\
\hline Collision risk warning from roadside units & $\mathrm{V} 2 \mathrm{~V}$ & Periodic/Event-driven & 10 & 100 \\
\hline \multicolumn{5}{|c|}{ ETSI - Traffic Management } \\
\hline Regulatory speed limit & $\mathrm{I} 2 \mathrm{~V}$ & Event-driven & $1-10$ & 500 \\
\hline Traffic light optimal speed advisory & $\mathrm{I} 2 \mathrm{~V}$ & Periodic & 2 & 100 \\
\hline Traffic information and recommended itinerary & $\mathrm{I} 2 \mathrm{~V}$ & Periodic & $1-10$ & 500 \\
\hline Enhanced route guidance and navigation & $\mathrm{I} 2 \mathrm{~V}$ & Periodic/Event-driven & 1 & 500 \\
\hline Intersection management & $\mathrm{I} 2 \mathrm{~V}$ & Periodic & 1 & 500 \\
\hline Cooperative flexible lane change & $\mathrm{I} 2 \mathrm{~V} / \mathrm{V} 2 \mathrm{~V}$ & Periodic/Event-driven & 1 & 500 \\
\hline Limited access warning & $\mathrm{I} 2 \mathrm{~V} / \mathrm{V} 2 \mathrm{~V}$ & Periodic/Event-driven & $1-10$ & 500 \\
\hline Electronic toll collect & $\mathrm{I} 2 \mathrm{~V} / \mathrm{V} 2 \mathrm{I}$ & Periodic/Event-driven & 1 & 200 \\
\hline Cooperative adaptive cruise control & $\mathrm{V} 2 \mathrm{X}$ & Periodic & 2 & 100 \\
\hline Highway platooning & $\mathrm{V} 2 \mathrm{X}$ & Periodic & 2 & 100 \\
\hline \multicolumn{5}{|c|}{ ETSI - Infotainment } \\
\hline Point of interest notification/Automatic access & $\mathrm{I} 2 \mathrm{~V} / \mathrm{V} 2 \mathrm{I}$ & Periodic/Event-driven & 1 & 500 \\
\hline Local electronic commerce/Instant messaging & $\mathrm{I} 2 \mathrm{~V} / \mathrm{V} 2 \mathrm{I}$ & Periodic/Event-driven & 1 & 500 \\
\hline Car rental/sharing assignment/reporting & I2V/V2I & Periodic/Event-driven & 1 & 500 \\
\hline Media downloading/Map download and update & $\mathrm{I} 2 \mathrm{~V} / \mathrm{V} 2 \mathrm{I}$ & Periodic/Event-driven & 1 & 500 \\
\hline Ecological/economical drive & $\mathrm{I} 2 \mathrm{~V} / \mathrm{V} 2 \mathrm{I}$ & Periodic/Event-driven & 1 & 500 \\
\hline Personal data synchronization/Vehicle relation management & $\mathrm{I} 2 \mathrm{~V} / \mathrm{V} 2 \mathrm{I}$ & Periodic/Event-driven & 1 & 500 \\
\hline SOS service/Stolen vehicle alert & $\mathrm{I} 2 \mathrm{~V} / \mathrm{V} 2 \mathrm{I}$ & Periodic/Event-driven & 1 & 500 \\
\hline Remote diagnosis and just in time repair notification & $\mathrm{I} 2 \mathrm{~V} / \mathrm{V} 2 \mathrm{I}$ & Periodic/Event-driven & 1 & 500 \\
\hline Vehicle data collect for product life cycle management & $\mathrm{I} 2 \mathrm{~V} / \mathrm{V} 2 \mathrm{I}$ & Periodic/Event-driven & 1 & 500 \\
\hline Insurance and financial Services & $\mathrm{I} 2 \mathrm{~V} / \mathrm{V} 2 \mathrm{I}$ & Periodic/Event-driven & 1 & 500 \\
\hline Fleet management/Loading zone management & I2V/V2I & Periodic/Event-driven & 1 & 500 \\
\hline Vehicle software/data provisioning and update & I2V/V2I & Periodic/Event-driven & 1 & 500 \\
\hline Vehicle and RSU data calibration & $\mathrm{I} 2 \mathrm{~V} / \mathrm{V} 2 \mathrm{I}$ & Periodic/Event-driven & 1 & 500 \\
\hline \multicolumn{5}{|c|}{ 3GPP - Safety } \\
\hline Forward collision warning & $\mathrm{V} 2 \mathrm{~V}$ & Periodic & 10 & 100 \\
\hline Control loss warning & $\mathrm{V} 2 \mathrm{~V}$ & Periodic & 10 & 100 \\
\hline V2V use case for emergency vehicle warning & $\mathrm{V} 2 \mathrm{~V}$ & Periodic & 10 & 100 \\
\hline V2V/V2I emergency stop use case & $\mathrm{V} 2 \mathrm{~V}$ & Periodic & 10 & 100 \\
\hline Queue warning & $\mathrm{V} 2 \mathrm{~V}$ & Periodic & N/A & 100 \\
\hline Warning to pedestrian against pedestrian collision & $\mathrm{V} 2 \mathrm{~V}$ & Periodic & N/A & N/A \\
\hline Vulnerable road user safety & $\mathrm{V} 2 \mathrm{~V}$ & Periodic & 1 & 100 \\
\hline \multicolumn{5}{|c|}{ 3GPP - Traffic Management } \\
\hline Cooperative Adaptive Cruise Control & $\mathrm{V} 2 \mathrm{~V}$ & Periodic/Event-driven & 1 & 1000 \\
\hline Traffic Flow Optimisation & $\mathrm{V} 2 \mathrm{~V}$ & Periodic / Event-driven & 0.1 & 1000 \\
\hline Automated Parking System & $\mathrm{V} 2 \mathrm{X}$ & Event-driven & N/A & 100 \\
\hline \multicolumn{5}{|c|}{ 3GPP - Infotainment } \\
\hline V2V message transfer under operator control & $\mathrm{V} 2 \mathrm{~V}$ & Event-driven & N/A & N/A \\
\hline
\end{tabular}

high reliability also in high density scenarios will be requested, and actual systems cannot satisfy all these requirements, since they are designed with the human user in mind. Some solutions to these limitation are addressed by $5 \mathrm{G}$ systems, as detailed in the next sections.

\section{5G VEHICULAR NETWORKS}

\section{A. 5G Requirements}

To face the requirements mentioned in Section III and especially those envisioned by new applications and selfdriving cars, based on $\mathrm{V} 2 \mathrm{~V}$ communication with real-time constraints, vehicles require new levels of connectivity and intelligence. 


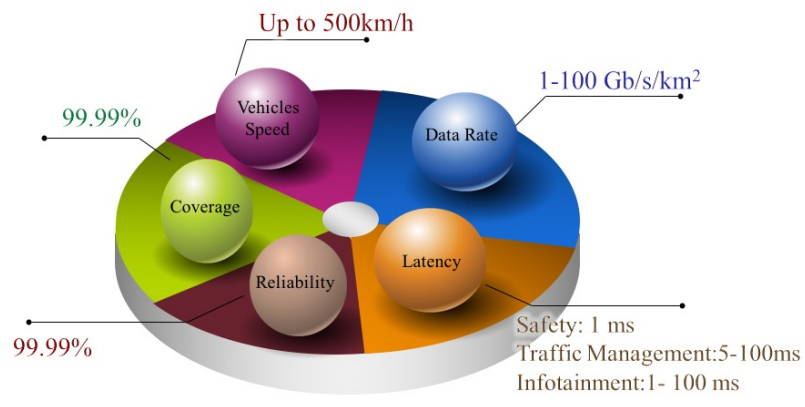

Fig. 1. Specifications for $5 \mathrm{G}$ vehicular networks.

The answer to this new challenging scenarios can be given by $5 \mathrm{G}$ systems, that foresee everything connected to everything else and able to cope with the users' demand. 5G systems will not only have to surpass previous generations in requirements such as capacity and latency, but they also have to address new applications and usage scenarios with very different requirements on capacity, reliability, and latency [23]-[25], such as augmented reality, see through technology, and, of course, autonomous vehicles.

In general, 5G vehicular networks will be based on the integration of flexible and reconfigurable on-the-fly radio interfaces, so that the following performance (also depicted in Fig. 1) could be provided [26]:

- Higher throughput: 1000 times higher mobile data volume per area and 10 to 100 times higher data rate. In addition, the possibility of sharing the same radio resources among users communicating through the infrastructure and $\mathrm{V} 2 \mathrm{~V}$ users, can improve the spectrum usage and the overall system capacity.

- Lower latency: 5 times reduced end-to-end latency. In $\mathrm{V} 2 \mathrm{~V}$ communications, the end-to-end latency may be reduced also exploiting short distances and no involvement of the infrastructure.

- Ultra-high reliability that can be obtained through V2V extended network coverage and through the exploitation of $\mathrm{V} 2 \mathrm{~V}$ network as fall back solution in the absence of the infrastructure.

- Higher connectivity density: 10 to 100 times higher number of connected devices;

- Higher mobility: up to $500 \mathrm{~km} / \mathrm{h}$;

- Full coverage.

The 5GPP roadmap indicates the requirements in terms of latency, which must be below $5 \mathrm{~ms}$, and density, which must be up to 100 devices $/ \mathrm{m}^{2}$, along with tight constraints on territory and users coverage even in areas without network coverage due to shadowing or other obstructions, by using, for example, relaying signals between vehicles [1]. 5G connectivity will allow vehicles to act as mobile hotspots, redistributing Internet connectivity to nearby users as they travel [9].

The 5GPP METIS project indicates some of the possible scenarios of the $5 \mathrm{G}$ vehicular network and suggests general requirements for safety-related applications in terms of end- to-end latency, beacon periodicity, reliability, communication range, positioning accuracy and experienced throughput, without identifying any specific use cases [27]:

- End-to-end Latency: $5 \mathrm{~ms}$;

- Beacon periodicity: $10 \mathrm{~Hz}$;

- Reliability: 99.999\%;

- Communication range: up to $1 \mathrm{Km}$;

- Positioning accuracy: less than $0.5 \mathrm{~m}$.

\section{B. 5G Radio Access}

The variety of services and the number of different requirements let a single radio interface not completely adequate to fit all the solutions. We expect that $5 \mathrm{G}$ systems will provide flexible and scalable radio interfaces to tailor the system configuration to the required service [28]. One of the main challenging problems is the massive access, hence the efficient handling of a huge number of users, through new MAC solutions.

Among the candidate 5G access technologies, we can cite:

- orthogonal frequency division multiple access (OFDMA), already exploited, for example, in the downlink of LTE;

- SC-FDMA, which is similar to OFDMA, but more robust against non linearities since complex symbols are transmitted sequentially rather than in parallel. SC-FDMA is foreseen by 3GPP Release 14 for V2V communications, as described in Section II;

- filtered-bank based multi carrier (FBMC), where the signals on the single subcarriers are independently filtered with orthogonal pulses;

- universal filtered orthogonal frequency division multiplexing (UF-OFDM), where sub-bands, made of a minimum number of sub-carriers, are filtered;

- non-orthogonal multiple access (NOMA) allows to multiplex multiple users in the power domain, by exploiting self-interference cancellation techniques at the receiver. This way, the number of users is not bounded by the number of orthogonal resources and the number of connected device can be doubled or tripled with respect to previous access techniques;

- sparse code multiple access (SCMA), another non orthogonal multiple access technique which allows users multiplexing in the code and power domains, over the same time-frequency resources.

To provide reliable V2X communications, it is desirable to have a flexible MAC scheme to cope with varying degrees of network connectivity. Hence, in addition to the above listed MAC access techniques, an initial phase of random access to sense the medium before resource assignment, has to be also considered [28].

\section{RADIO ACCESS EFFICIENCY TOWARDS 5G V2V}

We now focus on beaconing for cooperative awareness and we compare legacy LTE with infrastructure (LTE-V2I) with the LTE-V2V of Release 14 and further enhancements that are still debated. With beaconing we mean the periodic transmission, from each vehicle to all its neighbors within a 
defined range, hereafter called awareness range, of a message informing about its characteristics, position and movement. Such service is at the basis of all vehicular applications, since it allows being aware of the vehicles moving nearby.

\section{A. Compared Cases and Output Figures}

Specifically, the cases that are compared hereafter are:

- LTE-V2I standard: based on basic LTE (Release 8), each vehicle sends its beacon in the uplink to the network and the network retransmits a copy to all the vehicles in its awareness range; in this case, the bottleneck is in the downlink, since a number of copies must be transmitted per each vehicle.

- LTE-V2I with eMBMS: exploiting the eMBMS feature added in Release 9, the network broadcasts each beacon to all the vehicles in the cell, without the need to generate more copies; in this case, the bottleneck moves to the uplink, due to the lower transmission power available at the vehicles.

- $\boldsymbol{L T E}-\boldsymbol{V} 2 \boldsymbol{V}$ : thanks to the V2V addition in Release 14, the vehicles directly communicate to each other, without the need of exchanging messages with the e-NodesB; since each vehicle either transmits or receives in a subframe, the number of vehicles reciprocally communicating is upper bounded by the number of subframes per beacon period [29].

- LTE-V2V with short subframe (set to half the standard duration): as a $5 \mathrm{G}$ feature, the duration of a subframe is supposed to be halved; in such case, the maximum number of vehicles that can reciprocally communicate doubles.

- $\boldsymbol{L T E}-\boldsymbol{V} 2 \boldsymbol{V}$ with $\boldsymbol{F D}$ radios: as another $5 \mathrm{G}$ feature, the use of FD radios is supposed; with FD radios, each vehicle becomes able to concurrently transmit and receive during the same subframe, thus increasing the resources that can be exploited for beaconing.

As output metrics, we evaluate

1) the success probability of the allocation $P_{\mathrm{S}}$ and

2) the efficiency $\eta$ as [28]

$$
\eta=\frac{P_{\mathrm{S}} \cdot N_{\mathrm{U}}}{N_{\mathrm{R}}}
$$

where $N_{\mathrm{U}}$ is the average number of users in the cell (concurrently accessing the same resources) and $N_{\mathrm{R}}$ is the number of resources for beaconing in terms of maximum dedicated resource blocks per frame.

\section{B. Scenario, Notations and Assumptions}

Having in mind to focus on a simple and tractable example, we assume a highway segment covered by a single cell of radius $r_{\mathrm{c}}$, where vehicles are Poisson distributed with density $\rho$. Each vehicle is assumed to transmit a beacon of $B$ bytes every beaconing interval $T_{\mathrm{B}}$ (i.e., the beacon frequency is $f_{\mathrm{B}}=1 / T_{\mathrm{B}}$ ). The beacon is relevant for those vehicles that are in the awareness range $r_{\mathrm{aw}}$.
TABLE II

MAIN SETTINGS.

\begin{tabular}{|c|c|}
\hline Parameter (Symbol) & Value \\
\hline Common & \\
\hline Number of RBs dedicated to the service per frame $\left(N_{R}\right)$ & 408 \\
\hline Beacon frequency $\left(f_{\mathrm{B}}\right)$ & $10 \mathrm{~Hz}$ \\
\hline Beacon size $(B)$ & 100 \\
\hline Equivalent radiated power & $\begin{array}{l}\text { Vehicles: } 23 \mathrm{dBm} \\
\text { e-NodesB: } 33 \mathrm{dBm}\end{array}$ \\
\hline Antenna gain at the receiver & $3 \mathrm{~dB}$ \\
\hline Path loss at $1 \mathrm{~m}$ at $5.9 \mathrm{GHz}$ & $47.86 \mathrm{~dB}$ \\
\hline Loss exponent & 2.75 \\
\hline Noise power over an RB & $\begin{array}{l}\text { Vehicles: }-110 \mathrm{dBm} \\
\text { e-NodesB: }-115 \mathrm{dBm}\end{array}$ \\
\hline Cell range $\left(r_{\mathrm{c}}\right)$ & $500 \mathrm{~m}$ \\
\hline V2I unicast & \\
\hline MCS (downlink) & 17 \\
\hline Modulation & 16-QAM \\
\hline Coding rate & \\
\hline Maximum beacons per beacon period in downlink ( $\left.n_{\text {DL-V2I }}\right)$ & 340 \\
\hline V2I broadcast & \\
\hline MCS (downlink) & 11 \\
\hline Modulation & 16-QAM \\
\hline Coding rate & 0.30 \\
\hline Maximum beacons per beacon period in uplink ( $n_{\text {UL-V2I }}$ ) & 200 \\
\hline $\mathrm{V} 2 \mathrm{~V}$ & \\
\hline MCS (downlink) & 2 \\
\hline Modulation & 16-QAM \\
\hline Coding rate & 0.20 \\
\hline Maximum beacons per beacon period in uplink $\left(n_{\mathrm{V} 2 \mathrm{~V}}\right)$ & 100 \\
\hline Reuse distance & $198 \mathrm{~m}$ \\
\hline V2V with half duration subframe & \\
\hline MCS (downlink) & 5 \\
\hline Modulation & 16-QAM \\
\hline Coding rate & 0.40 \\
\hline Maximum beacons per beacon period in uplink ( $\left.n_{\mathrm{V} 2 \mathrm{~V} \text {-shsf }}\right)$ & \\
\hline Reuse distance & $246 \mathrm{~m}$ \\
\hline$V 2 V$ with $F D$ radios & \\
\hline Self interference cancellation (FD) & $-110 \mathrm{~dB}$ \\
\hline MCS (downlink) & \\
\hline Modulation & 16-QAM \\
\hline Coding distance & 0.47 \\
\hline Maximum beacons per beacon period in uplink $\left(n_{\mathrm{V} 2 \mathrm{~V}-\mathrm{FD}}\right)$ & 250 \\
\hline Reuse range & $287 \mathrm{~m}$ \\
\hline
\end{tabular}

In the following, we denote the average number of vehicles per cell as $n_{\mathrm{v}}$ and we use the function

$$
F_{\mathrm{P}}(n, \rho, \ell) \triangleq 1-\frac{\Gamma(n, \rho \cdot \ell)}{\Gamma(n)}
$$

where $\Gamma(\cdot)$ is the Euler Gamma function and $\Gamma(\cdot, \cdot)$ the incomplete Euler Gamma function.

In all cases, calculations are based on the maximum number of beacons that can be allocated per beacon period (in downlink, uplink or $\mathrm{V} 2 \mathrm{~V}$, depending on the case), which has been obtained following the 3GPP documentation and the approach detailed in [29].

The main settings are summarized in Table II and the adopted modulation and coding scheme (MCS) is always the one that guarantees the cell coverage using the least resource blocks per beacon.

\section{LTE for V2I}

If vehicles were equipped with LTE before Release 14, beacons would need to be sent first to some network entity through V2I in the uplink, and then forwarded back from the same entity to the neighbor vehicles through unicast or broadcast $\mathrm{I} 2 \mathrm{~V}$ in the downlink. 
Unicast. In the case of standard unicast transmissions, the success probability of the allocation $P_{\mathrm{S} \text {-V2I-u }}$ corresponds to the probability that resources are enough to allocate all the copies of the beacons to be forwarded in downlink to the neighbors of each vehicle. Referring to the calculations in [13] and approximating with $n_{\mathrm{v}} \cdot r_{\mathrm{aw}} / r_{\mathrm{c}}$ the average number of neighbors in the awareness range of each vehicle, it is

$$
P_{\text {S-V2I-u }}=1-F_{P}\left(\left[\sqrt{n_{\text {DL-V2I }} \frac{r_{\mathrm{c}}}{r_{\text {aw }}}}\right\rceil, \rho, 2 r_{\mathrm{c}}\right)
$$

where $n_{\text {DL-V2I-u }}$ is the maximum number of beacons that can be allocated per beacon period in this case.

Broadcast. In the case of broadcast transmission in downlink, the success probability of the allocation $P_{\mathrm{S}-\mathrm{V} 2 \mathrm{I}-\mathrm{b}}$ corresponds to the probability that resources are enough to allocate the beacons to be collected from all vehicles in uplink [13],

$$
P_{\mathrm{S}-\mathrm{V} 2 \mathrm{I}-\mathrm{b}}=1-F_{P}\left(n_{\mathrm{UL}-\mathrm{V} 2 \mathrm{I}}, \rho, 2 r_{\mathrm{c}}\right)
$$

where $n_{\mathrm{UL}-\mathrm{V} 2 \mathrm{I}}$ is the maximum number of beacons that can be allocated per beacon period in this case.

\section{LTE-V2V as in Release 14}

Beacons are sent directly from each vehicle to its neighbors using V2V. In this case, the same resource block (RB) can be used by more vehicles in the cell if they are far enough from each other. We denote as reuse distance $r_{\text {reuse }}$, the minimum distance at which a vehicle can transmit using the same RB of another transmitting vehicle without causing errors due to interference towards any of the vehicles in the awareness range of the transmitting one.

Following [13], the success probability of the allocation $P_{\mathrm{S}-\mathrm{V} 2 \mathrm{~V}}$, corresponding to the probability that the allocation succeeds without any blocked vehicle, can be calculated as

$$
P_{\mathrm{S}-\mathrm{V} 2 \mathrm{~V}}=\left[1-F_{\mathrm{P}}\left(\left\lceil n_{\mathrm{V} 2 \mathrm{~V}}\right\rceil, \rho, r_{\text {reuse }}\right)\right]^{\left\lceil\frac{2 r_{\mathrm{c}}}{r_{\text {reuse }}}\right\rceil}
$$

where $n_{\mathrm{V} 2 \mathrm{~V}}$ is the maximum number of beacons that can be allocated per beacon period in this case.

Please recall that, as already discussed, with standard half duplex (HD) radios each vehicle can either transmit or receive in a subframe, thus $n_{\mathrm{V} 2 \mathrm{~V}}$ is upper bounded by $T_{\mathrm{B}}$ divided by $1 \mathrm{~ms}$.

\section{E. LTE-V2V with Half Duration Subframes}

Projecting to $5 \mathrm{G}$ vehicular networks, it is considered a viable option to halve the subframe duration to reduce the latency. Looking at beaconing, this modification would allow more beacons to be allocated in a beacon period, since the upper bound to the maximum number of beacons that can be allocated per beacon period $n_{\mathrm{V} 2 \mathrm{~V} \text {-shsf }}$ increases to $T_{\mathrm{B}}$ divided by $0.5 \mathrm{~ms}$.

The success probability $P_{\mathrm{S}-\mathrm{V} 2 \mathrm{~V}-\text { shsf }}$ can be again calculated using (5), substituting $n_{\mathrm{V} 2 \mathrm{~V}}$ with $n_{\mathrm{V} 2 \mathrm{~V} \text {-shsf }}$.

When referring to LTE-V2V with half duration subframes, we suppose that the ODFM symbol duration halves compared to normal subframes, while subcarrier spacing doubles, so that the number of RBs per frame and the number of data

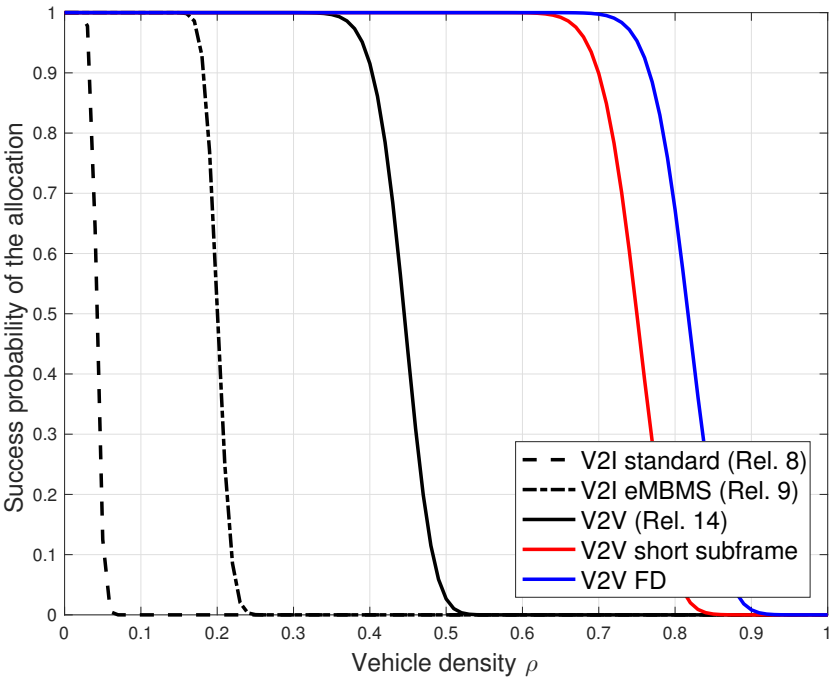

Fig. 2. Success probability of beacon allocation in LTE towards 5G.

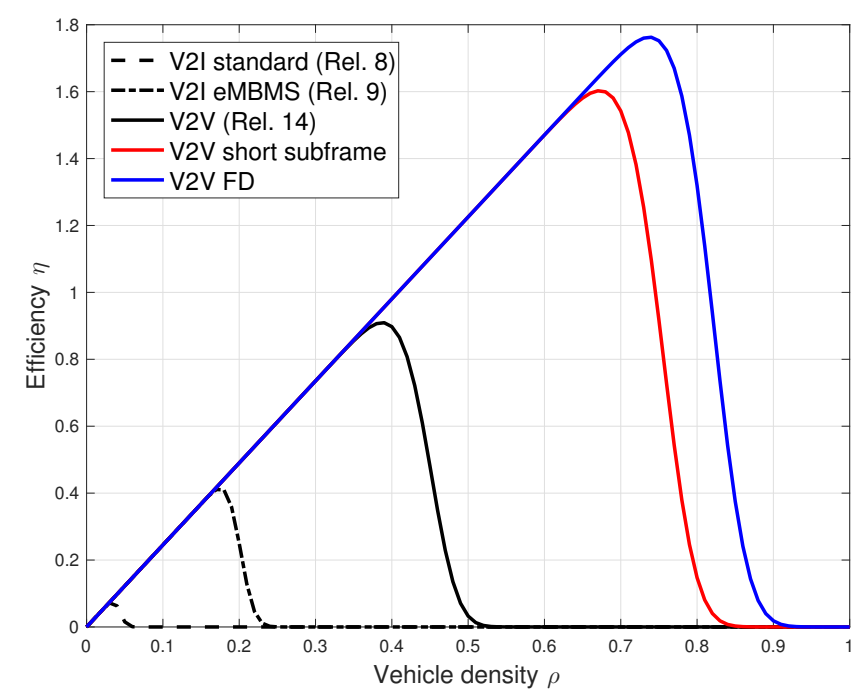

Fig. 3. Efficiency of LTE to support V2V beaconing towards 5G.

symbols per RB remain the same. With these assumptions, the reuse range adopting half duration subframes increases since transmissions are performed over a larger bandwidth and are thus subject to higher noise.

\section{F. LTE-V2V with FD radios}

Another feature foreseen in $5 \mathrm{G}$ vehicular networks is the adoption of FD radios. Their use for beaconing would allow the allocation of beacons per subframe also sent by vehicles in the reciprocal range, thus exploiting also the frequency domain that the HD does not allow to efficiently use [13].

The success probability $P_{\mathrm{S}-\mathrm{V} 2 \mathrm{~V}-\mathrm{FD}}$ can be again calculated using (5), substituting $n_{\mathrm{V} 2 \mathrm{~V}}$ with the maximum number of beacons that can be allocated per beacon period with FD radios $n_{\mathrm{V} 2 \mathrm{~V}-\mathrm{FD}}$. In this case, the reuse range increases compared to normal $\mathrm{V} 2 \mathrm{~V}$, due to the residual self interference. 


\section{G. Numerical Results}

In Figs. 2 and 3, the success probability of the allocation $P_{\mathrm{S}}$ and the efficiency $\eta$ as defined in (1), are plotted varying the vehicle density $\rho$ for the various cases. As one can see, by using standard V2I with unicast transmission, the beaconing service can be supported only under low vehicle densities (less than one vehicle every 20-30 meters on a highway), with an $\eta$ that reaches at most 0.07 vehicles per resource block per cell. Exploiting eMBMS to distribute the beacons in downlink in broadcast allows to significantly improve the performance of the network, reaching a maximum $\eta$ of 0.41 , which is however still far less than that allowed by V2V. As a first step on the road to $5 \mathrm{G}$, the adoption of LTE-V2V as in Release 14 brings to double the efficiency compared to V2I with broadcast, with a maximum $\eta$ of 0.91 . And the discussed features foreseen for $5 \mathrm{G}$ allow to further increase such value to 1.60 thanks to half subframes and even 1.76 thanks to FD radios.

\section{CONCLUSiON}

The interest in connected vehicles is increased and wireless access is made possible by a variety of technologies. Some of these are already commercial, such as cellular communication for V2I communications or short range communications based on IEEE 802.11p. But new requirements and applications are around the corner and actual technologies cannot satisfy them. LTE-V2V represents a step toward 5G and example performance are provided in this paper. However, it is probable that the future standard of vehicular communications will be a mashup of WAVE/IEEE 802.11p in the US and ETSI 5G for C-ITS in Europe with 5G, by adopting the first technology for wireless access signaling and $5 \mathrm{G}$ for data transmission and service provisioning.

\section{ACKNOWLEDGMENT}

This work has been carried out in the framework of the project IMATISSE (Inundation Monitoring and Alarm Technology In a System of SystEms), which is funded by the Region Picardie, France, through the European Regional Development Fund (ERDF).

\section{REFERENCES}

[1] 5g empowering vertical industries (february 2016). Accessed on March 2017. [Online]. Available: https://5g-ppp.eu/roadmaps/

[2] Volvo cars new xc60 suv will automatically steer you out of trouble (february 2017). Accessed on March 2017. [Online]. Available: https://www.media.volvocars.com/global/en$\mathrm{gb} / \mathrm{media} /$ pressreleases/204531/volvo-cars-new-xc60-suv-willautomatically-steer-you-out-of-trouble

[3] "Standard for information technology- telecommunications and information exchange between systems- local and metropolitan area networksspecific requirements part 11 - amendment 6: Wireless access in vehicular environment," IEEE, 2010.

[4] A. Bazzi, B. M. Masini, A. Zanella, and G. Pasolini, "Vehicle-tovehicle and vehicle-to-roadside multi-hop communications for vehicular sensor networks: Simulations and field trial," in 2013 IEEE International Conference on Communications Workshops (ICC), June 2013, pp. 515520.

[5] 3GPP: Initial cellular V2X standard completed. Accessed on January 2017. [Online]. Available: http://www.3gpp.org/news-events/3gppnews/1798-v2x_r14
[6] H. Seo, K. D. Lee, S. Yasukawa, Y. Peng, and P. Sartori, "LTE evolution for vehicle-to-everything services," IEEE Communications Magazine, vol. 54, no. 6, pp. 22-28, June 2016.

[7] S. h. Sun, J. 1. Hu, Y. Peng, X. m. Pan, L. Zhao, and J. y. Fang, "Support for vehicle-to-everything services based on LTE," IEEE Wireless Communications, vol. 23, no. 3, pp. 4-8, June 2016.

[8] A. Festag, "Standards for vehicular communication-from ieee $802.11 \mathrm{p}$ to 5g," e \& i Elektrotechnik und Informationstechnik, vol. 132, no. 7, pp. 409-416, 2015. [Online]. Available: http://dx.doi.org/10.1007/s00502015-0343-0

[9] A. Nordrum, "Autonomous Driving Experts Weigh 5G Cellular Network Against Dedicated Short Range Communications," IEEE Spectrum, Cars That Think, May 2016.

[10] J. Yang, B. Pelletier, and B. Champagne, "Enhanced autonomous resource selection for lte-based v2v communication," in 2016 IEEE Vehicular Networking Conference (VNC), Dec 2016, pp. 1-6.

[11] J. Lee and D. Chang, "Performance comparison of V2V communication based on LTE-D2D with cooperative schemes," in 2016 International Conference on Information and Communication Technology Convergence (ICTC), Oct 2016, pp. 921-925.

[12] O. Y. Kwon, R. Song, Y. Z. Ma, and B. S. Kim, "Integrated MIMO antennas for LTE and V2V applications," in 2016 URSI Asia-Pacific Radio Science Conference (URSI AP-RASC), Aug 2016, pp. 1057-1060.

[13] A. Bazzi, B. M. Masini, and A. Zanella, "Performance analysis of V2V beaconing using LTE in direct mode with full duplex radios," IEEE Wireless Communications Letters, vol. 4, no. 6, pp. 685-688, Dec. 2015.

[14] A. Bazzi, B. M. Masini, A. Zanella, and I. Thibault, "Beaconing from connected vehicles: Ieee $802.11 \mathrm{p}$ vs. 1te-v2v," in 2016 IEEE 27th Annual International Symposium on Personal, Indoor, and Mobile Radio Communications (PIMRC), Sept 2016, pp. 1-6.

[15] A. M. Vegni and E. Natalizio, "A hybrid (n/m)cho soft/hard vertical handover technique for heterogeneous wireless networks," Elsevier $\mathrm{Ad}$ Hoc Networks, vol. 14, pp. 51-70, 2014.

[16] A. M. Vegni, A. Stramacci, and E. Natalizio, "Opportunistic clusters selection in a reliable enhanced broadcast protocol for vehicular ad hoc networks," in Wireless On-demand Network Systems and Services (WONS), 2013 10th Annual Conference on, 2013, pp. 95-97.

[17] A. Bazzi, B. M. Masini, A. Zanella, and G. Pasolini, "IEEE 802.11p for cellular offloading in vehicular sensor networks," Computer Communications, vol. 60, pp. 97 - 108, 2015.

[18] "IEEE standard for local and metropolitan area networks-part 15.7: Short-range wireless optical communication using visible light," IEEE Std 802.15.7-2011, pp. 1-309, Sep. 2011.

[19] 3GPP, "Study on LTE support for V2X services," TR 22.885 V14.0.0, Dec. 2015.

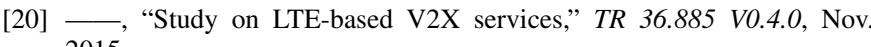
2015.

[21] G. A. A. 5GAA, "The Case for Cellular V2X for Safety and Cooperative Driving," 5GAA White Paper, 2016.

[22] "Intelligent transport systems (ITS); vehicular communications; basic set of applications; definitions," TR 102.638 V1.1.1, June 2009.

[23] H. Cao, S. Gangakhedkar, A. R. Ali, M. Gharba, and J. Eichinger, "A 5G V2X testbed for cooperative automated driving," in 2016 IEEE Vehicular Networking Conference (VNC), Dec 2016, pp. 1-4.

[24] H. Droste, G. Zimmermann, M. Stamatelatos, N. Lindqvist, O. Bulakci, J. Eichinger, V. Venkatasubramanian, U. Dotsch, and H. Tullberg, "The metis $5 \mathrm{~g}$ architecture: A summary of metis work on $5 \mathrm{~g}$ architectures," in 2015 IEEE 81st Vehicular Technology Conference (VTC Spring), May 2015, pp. $1-5$.

[25] J. Gozalvez, "Tentative 3gpp timeline for $5 \mathrm{~g}$ [mobile radio]," IEEE Vehicular Technology Magazine, vol. 10, no. 3, pp. 12-18, Sept 2015.

[26] H. Tullberg, P. Popovski, Z. Li, M. A. Uusitalo, A. Hoglund, O. Bulakci, M. Fallgren, and J. F. Monserrat, "The metis 5g system concept: Meeting the $5 \mathrm{~g}$ requirements," IEEE Communications Magazine, vol. 54, no. 12 , pp. 132-139, December 2016.

[27] METIS-ii: Mobile and wireless communications enablers for twentytwenty (2020) information society-ii. Accessed on March 2017. [Online]. Available: https://5g-ppp.eu/metis-ii/

[28] A. Osseiran, J. F. Monserrat, and P. Marsch, $5 G$ Mobile and Wireless Communications Technologies. Cambridge University Press, 2016.

[29] A. Bazzi, B. M. Masini, and A. Zanella, "How many vehicles in the LTE-V2V awareness range with half or full duplex radios?" in 15th edition of International Conference on Intelligent Transport Systems Telecommunications (ITST), May 2017. 